Präv Gesundheitsf 2014 · 9:157-158

DOI 10.1007/s11553-014-0447-0

Online publiziert: 10. Juli 2014

๑) Springer-Verlag Berlin Heidelberg 2014

\title{
Patrick Timpel
}

Forschungsverbund Public Health Sachsen und Sachsen-Anhalt,

Medizinische Fakultät der TU Dresden, Dresden, Deutschland

\section{Editorial}

zu konzipieren und infolgedessen Monitoring- und Frühwarnsysteme zu entwickeln.

Das vorliegende Schwerpunktheft verbindet die Perspektiven der Medizin und Pharmakologie mit Analysen der Siedlungswasserwirtschaft und Mikrobiologie. Es werden ebenso geoinformationstechnische Fragestellungen wie auch kommunikations- und sozialwissenschaftliche Herausforderungen der Krisen- und Risikokommunikation erörtert. Dabei wird der Spagat zwischen Kläranlagenpraxis, Siedlungswasserwirtschaft und dem medizinisch verantwortungsvollen Umgang mit Antibiotika im ambulanten wie stationären Gesundheitssektor gewagt. Aus diesem Anspruch heraus wurde das Schwerpunktheft in verschiedene Themenfelder gegliedert:

- Grundlagen

- Antibiotikaanalytik im Abwasser und anderen Matrizes

- Detektion und Umgang mit Antibiotikaresistenzen

- Modellbildung und Datenmanagement

- Risikokommunikation zur Schaffung von Transparenz im Wasserkreislauf.

Im ersten Beitrag Antibiotika und Resistenzentwicklung werden die mikrobiologischen Grundlagen und Wirkmechanismen der wichtigsten Antibiotikagruppen exemplarisch betrachtet. Joachim Fauler beschreibt die ebenso aufwändige wie kostspielige Entwicklung neuer Antibiotika und die rückläufige Anzahl an Neuzulassungen.

$\mathrm{Um} \mathrm{zu}$ analysieren, in welchen Konzentrationen Antibiotika ins Abwasser gelangen und wie sich dies auf die dortige Bakteriengemeinschaft auswirkt, werden im Rahmen des Projektes ANTI-Resist an verschiedenen Punkten in der Kanali- sation des Stadtgebietes Dresden und der Kläranlage Dresden-Kaditz Proben genommen. Die im Projekt entwickelte und validierte Analysemethode ermöglicht es Antibiotikakonzentrationen zu bestimmen. Möglichkeiten und Grenzen dieses Antibiotikamonitorings in verschiedenen Abwässern werden von Reinhard Oertel analysiert. Sara Schubert zeigt anschließend welche der am häufigsten verschriebenen Antibiotika im urbanen Abwassersystem Dresdens akkumulieren. Neben der Akkumulation an Feststoffen, wie Sedimenten und Klärschlämmen, wird die Stabilität der Antibiotika beschrieben.

Die Beiträge des Themenfeldes Detektion und Umgang mit Antibiotikaresistenzen beschreiben das ernstzunehmende Problem der Entwicklung und Verbreitung von Antibiotikaresistenzen als eine neue Form der Umweltverschmutzung. Serena Caucci und Thomas Berendonk beleuchten die ökologischen und gesundheitlichen Folgeerscheinungen von Antibiotikaresistenzgenen in kommunalen Abwässern. Dabei wird gezeigt, dass Kläranlagen nicht nur als Reservoir sondern auch als Plattform zur Verbreitung von Resistenzgenen in die Umwelt anzusehen sind. Durch kulturbasierte und molekulare Methoden wird in diesem Beitrag der Frage nachgegangen, warum moderne Kläranlagen nicht dazu konzipiert sind, Resistenzgene gänzlich zu eliminieren. Anschließend skizziert Celia Manaia einen Überblick über den Stand der Wissenschaft bezüglich Antibiotikaresistenzen im Abwasser. In einer nachfolgenden Literaturstudie von Helmut Bürgmann mit besonderem Fokus auf die Schweiz wird deutlich, dass selbst Länder mit einem bereits etablierten Risikomanagement (Beispiel „MicroPoll“), zusätzlichen Reinigungsstufen bei der Abwasserbehandlung und niedrigerem Ver- 
schreibungsniveau von Antibiotika, mit nennenswerten Resistenzproblematiken zu kämpfen haben.

Als erster Beitrag des Themenfeldes Modellbildung und Datenmanagement betrachtet Viktoria Mühlbauer den humanen Antibiotikaverbrauch am Beispiel der Stadt Dresden. Auf Grundlage von Diagnose- und Verordnungsdaten der AOK PLUS (AOK Sachsen und Thüringen) sowie Lieferdaten der Krankenhausapotheken dreier Krankenhäuser Dresdens werden hier Verordnungsmuster sowie saisonale und altersspezifische Unterschiede identifiziert. Zusätzlich zu diesen zwei Datenquellen erfolgte eine Analyse der Antibiotikakonzentration im Zulauf der Kläranlage durch Conrad Marx und Volker Kühn. Diese betrachten die Wiederfindungsraten und das Transportverhalten der Antibiotikagruppen. Der Einfluss von Regenereignissen auf den Wasserund Stofftransport wird im nachfolgenden Beitrag von Thomas Käseberg und Kollegen analysiert. Luise Hutka, Daniel Kadner und Lars Bernard widmen sich der Entwicklung eines Eintrags- und Prognosemodells zur Abschätzung des zu erwartenden Antibiotika-Aufkommens. Dabei wurde ein Frühwarnsystem zur Vorhersage kritischer Zustände von Antibiotikaeinträgen konzipiert. Das ebenso entwickelte Geoportal visualisiert die Daten und Ergebnisse der Analysen in Form von Karten und Diagrammen.

Im Themenfeld Risikokommunikation zur Schaffung von Transparenz im Wasserkreislauf beschreibt Norbert Lucke den Aufbau und die Funktionsweise des Dresdner Abwassersystems. Marion Dreyer und Kollegen widmen sich im nachfolgenden Beitrag Risikokommunikation zu Arzneimitteln in Gewässern: Ein Balanceakt der Risikowahrnehmungsforschung. Der abschließende Artikel Krisen- und Risikokommunikation von Beate Renner und Martina Gamp setzt am Beispiel des Legionellenausbruchs in Warstein im Sommer 2013 an und zeigt welche unmittelbare Krisenkommunikation in Folge eines Schadenfalls einzuleiten ist.

In der Gesamtschau gibt das vorliegende Schwerpunktheft Antibiotika und Antibiotikareistenzen im urbanen Abwasser einen Einblick in die Folgen des großzügigen und unkritischen Einsatzes von
Antibiotika. Es dient der Wissensvermittlung und Sensibilisierung für die Zusammenhänge von Antibiotikaverschreibung, Resistenzentwicklung und Umweltrisiko. Der „schleichenden Gefahr“ der Verbreitung von Antibiotika und Antibiotikaresistenzen im Abwasser muss durch einen verantwortungsvollen Umgang mit Arzneimitteln begegnet werden. Dieses Arbeitsfeld steht erst am Anfang. Das Schwerpunktheft bietet jedoch einen fundierten Einblick in den aktuellen Stand der Wissenschaft zur Entwicklung eines Risikomanagements für den vorsorgenden Gesundheits- und Umweltschutz.

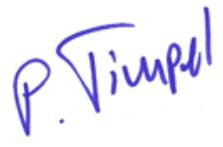

\section{Patrick Timpel}

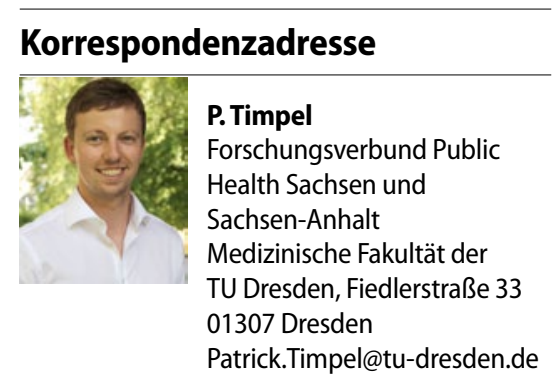

